\title{
Seguimiento de egresados de la licenciatura de la ENEO-UNAM formación y desempeño
}

\section{laboral (2003-2006)}

Mtra. Ma. Cristina Müggenburg Rodríguez V**Mtra. Iñiga Pérez Cabrera**,

Mtra. Elvia Leticia Ramírez Toríz***, Mtra. María Cristina Castañeda Godínez***

* Profesor de carrera T.C. Asociado "A" ENEO-UNAM, Coordinadora de la Maestría de la ENEO-UNAM.

** Profesor de carrera T.C. Asociado "C" ENEO-UNAM.

*** Profesor de carrera T.C. Asociado "B" ENEO-UNAM.

La ENEO-UNAM ha institucionalizado el proyecto seguimiento de egresados en el marco laboral, para identificar las mejores estrategias en la formación profesional que demanda más equilibrio social ante el desarrollo tecnológico de nuestro tiempo.

Visualizar su desempeño en el marco laboral y formativo a 3 años del termino de sus estudios realimentando el proceso formativo y orientándolos hacía el mercado laboral, dando respuesta a las necesidades sociales.

Estudio observacional, descriptivo, egresados de la Licenciatura en Enfermería y Obstetricia generación 2003, El registro en el sistema escolar correspondió a 132 casos, muestra probabilística, 41 exalumnos. Entrevista indirecta de 52 reactivos, orientados a características generales, formación académica, desempeño profesional, expectativas para el desarrollo profesional, opinión sobre reconocimiento y satisfacción profesional. Procesamiento de datos, con pruebas de estadística descriptiva e inferencial. Investigación de riesgo mínimo, confidencialidad de información ofrecida.

La demanda de egresados respecto a mejorar la enseñanza y práctica clínica. La contratación y ocupación de mejores categorías sigue favorecida con la titulación oportuna, el predominio del sector privado de contratación, en las actividades orientadas a la curación con desventaja en actividades de prevención, sin las cuales el sistema de salud no podrá darse abasto.

La tendencia positiva entre la formación académica y práctica del cuidado, propiciarán mayor congruencia entre formación y desempeño, esto permitirá mejorar impactos económicos, políticos y culturales de la globalización.

La proporción de desempleo es baja, comparada con otras áreas, pero la doble jornada y la baja remuneración son factores de alta preocupación para el gremio.

Palabras clave: egresados, formación profesional.

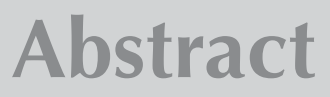

ENEO has instituted a program of follow-up with its graduates as they enter the workforce, in order to identify the most effective professional development strategies. This program seeks to asses the effectiveness of professional development in the modern high-tech workforce while attending to the needs of society.

ENEO will observe graduates' development for three years following graduation in order to provide each student with feedback over the course of their postgraduate development, coordinating this feedback with the needs of society. 
Observe ENEO graduates who completed their Bachelor's Degrees in 2003. School records indicate 132 such students. Our sample corresponds to 41 graduates. We conducted indirect interviews with 52 questions, focusing on general characteristics, academic formation, professional performance, professional development expectations, and each individual's own opinions regarding professional satisfaction and recognition. Afterwards, we conducted statistical and inferential analysis of the data. Low-risk investigation with confidentiality provided to participants.

Student needs pertaining to clinical theory and practice. The best professional opportunities continue to be available for those who complete their degrees; private sector opportunities continue to dominate the professional market; most professional opportunities focus on cures rather than prevention, a condition which places the health system at no small risk.

Good academic formation correlates positively with providing effective care, thus demonstrating that good preparation leads to good performance. This will allow for more economic, political and cultural impact in these times of globalization.

The unemployment rate is lower compared to other sectors of the economy, but the long hours and low salaries are a significant concern for healthcare workers.

Key words: graduates, professional performance.

\section{INTRODUCCIÓN}

La Escuela Nacional de Enfermería y Obstetricia(ENEO) de la UNAM, preocupada por la inserción y desempeño óptimo de sus egresados en el mercado laboral busca identificar las mejores estrategias para la formación profesional de sus alumnos, ubicados en un mundo que demanda más equilibrio social y a la vez más desarrollo tecnológico. Una de las estrategias que ha empleado es el seguimiento de sus egresados, buscando la valoración de la congruencia que se da entre la formación recibida y el desempeño laboral, ${ }^{\prime}$ con el fin de tomar decisiones en la planeación educativa de los programas que constituyen el Plan de Estudios de la Licenciatura. Este informe, corresponde a la duodécima generación de egreso, quienes finalizan su carrera en el 2003 y son encuestados en el 2006.

El propósito general, se enfocó a la visualización del desempeño profesional de los egresados de la ENEO en el marco laboral y formativo a los tres años de la terminación de sus estudios con el fin de retroalimentar el proceso formativo de la carrera y orientar a los alumnos y exalumnos para su inserción en el mercado laboral, buscando dar respuesta a las necesidades sociales. ${ }^{2}$

\section{MATERIAL Y MÉTODOS}

El presente estudio es observacional, descriptivo, de la población escolar de la Licenciatura en Enfermería que terminó su carrera en el 2003. La población escolarizada de Licenciados en Enfermería, registrada en el sistema escolar de la ENEO, correspondió a 132 casos, $^{3}$ a partir de lo cual se calculó una muestra de 41 exalumnos. Como en otros seguimientos, aún cuando se programó una muestra probabilística, hubo egresados que ofrecen su participación y aunque no estaban considerados en la muestra y fueron encuestados.

Los datos se recolectaron en el plantel en la mayoría de los casos, previa invitación a participar por vía telefónica, se aplicó una entrevista indirecta constituida por 52 reactivos, orientados a las características generales de la población de estudio, formación académica, desempeño profesional, expectativas para el desarrollo profesional, opinión sobre el reconocimiento profesional y satisfacción profesional. Las preguntas en su mayoría fueron cerradas, pero también hubo abiertas y mixtas. El procesamiento de datos se realizó utilizando principalmente pruebas de estadística descriptiva e inferencial. Esta investigación se considera con riesgo mínimo, pero se cuida la confidencialidad de la información ofrecida por los egresados.

\section{RESULTADOS}

La muestra estudiada correspondió a 41 egresados de la Licenciatura del sistema escolarizado, quienes terminaron sus estudios en el 2003, con el 100\% de créditos. 
La edad promedio fue de 28 años, encontrando al 61\% entre los 24 y los 27 años, al 24\% entre los 28 y los 30 años y el 15\% entre 31 y 42 años. El 78\% eran mujeres y el $22 \%$ hombres. El estado civil referido fue: $58 \%$ solteros, el $34 \%$ casados y el $8 \%$ en unión libre; del total de encuestados el 39\% tenía por lo menos un hijo, sin embargo algunos refieren dos o tres hijos. Respecto a su perfil académico, se puede mencionar lo siguiente: el promedio de calificación fue de 8.3 , el 76\% osciló entre 8.1 y 9.0, el 12\% estuvo por arriba de 9 y el $12 \%$ tuvo desde 7.6 hasta 8.0. En cuanto a la reprobación, el 58\% refirió haber reprobado durante su carrera por lo menos una materia y de estos menos de una tercera parte reprobó más de una materia. Entre las materias con más alto índice de reprobación se encontraron: Anatomía (43\%), Enfermería del Adolescente, Adulto y Anciano (13\%), Nutrición (8\%) y el resto quedó distribuido entre diversas materias, como Proceso Salud Enfermedad en el Niño, Enfermería del Niño y Ecología. (Cuadro No. 1).

\section{CUADRO NO. 1}

Materias reprobadas por los alumnos de la ENEO, egresados de la Licenciatura en 2003, encuestados a los tres años de su egreso.

2006

\begin{tabular}{|l|c|}
\hline \multicolumn{1}{|c|}{ Materia } & Porcentaje * \\
\hline Anatomía & $43 \%$ \\
\hline $\begin{array}{l}\text { Enfermería del Adolescente, del Adulto y del } \\
\text { Anciano }\end{array}$ & $13 \%$ \\
\hline Nutrición & $8 \%$ \\
\hline $\begin{array}{l}\text { Proceso Salud Enfermedad en el niño, Enfermería } \\
\text { del niño y Ecología }\end{array}$ & $36 \%$ \\
\hline
\end{tabular}

Fuente: Encuesta realizada a exalumnos de la Licenciatura del escolarizado que egresaron en 2003 de la ENEO. $n=41$

La opinión de los encuestados en cuanto a la calidad de la formación teórica recibida durante su carrera fue: el 83\% consideró que los conocimientos adquiridos apoyan "mucho" su desempeño profesional actual y el 17\% consideró que lo apoyan "regular". (Cuadro No. 2). Respecto la formación práctica recibida el 64\% de los egresados consideraron que las habilidades técnicas adquiridas habían apoyado "mucho" su desempeño, el 34\% que lo apoyan "regular" y el 2\% "poco" (Cuadro No. 3). En cuanto al Servicio Social el 21\% lo realizó en el Primer Nivel de Atención, el 18\% en el Segundo Nivel, el 33\% en el Tercer Nivel, el 13\% en Investigación, el 10\% en Docencia y el 5\% por artículo 91 y otros.

\section{CUADRO NO. 2}

Calidad de la formación teórica recibida durante la carrera, según opinión de los exalumnos de la ENEO, egresados de la licenciatura en 2003, encuestados a los 3 años de su egreso.

2006

\begin{tabular}{|l|c|}
\hline \multicolumn{1}{|c|}{$\begin{array}{c}\text { Apoyo de los conocimientos } \\
\text { para el desempeño }\end{array}$} & Porcentaje* \\
\hline Mucho & $83 \%$ \\
\hline Regular & $17 \%$ \\
\hline
\end{tabular}

Fuente: Misma del cuadro anterior. $n=41$

\section{CUADRO NO. 3}

Calidad de la formación práctica recibida durante la carrera, según opinión de los exalumnos de la ENEO, egresados de la licenciatura en 2003, encuestados a los 3 años de su egreso.

2006

\begin{tabular}{|c|c|}
\hline $\begin{array}{l}\text { Apoyo de la formación práctica para el } \\
\text { desempeño }\end{array}$ & Porcentaje* \\
\hline Mucho & $64 \%$ \\
\hline Regular & $34 \%$ \\
\hline Poco & $2 \%$ \\
\hline
\end{tabular}

Fuente: Misma del cuadro anterior $* n=41$

La proporción de titulados fue de 71\%; de estos, el 35\% tenía más de seis meses y hasta un año de haberse titulado, el 30\% tenía más de un año y menos de dos, otro $30 \%$ tenía seis meses o menos y el resto más de dos años (5\%). El 59\% afirma que los profesores propiciaron su titulación. Las opciones de titulación más seleccionadas fueron el EPAC y la tesis, ocupando el $41 \%$ y el $35 \%$ respectivamente, le siguió el PAE con un $15 \%$ y el resto seleccionó otra opción. Respecto a las que se encuentran en proceso de titulación, la mayoría está esperando para presentar el siguiente EPAC.

En cuanto a su situación laboral el 90\% está trabajando actualmente o ha estado ya formando parte de la fuerza de trabajo; el 10\% que no ha trabajado en su mayoría lo atribuye a falta de oportunidad en el mercado laboral, seguido de: baja remuneración, motivos familiares, bajo 
reconocimiento y problemas de género. El 62\% de los que laboran tiene por lo menos un año en el empleo, dos terceras partes tiene entre uno y dos años y la tercera parte tiene más de dos años; 38\% de tiene menos de un año laborando. Las instituciones contratantes en orden de frecuencia fueron: el sector privado, incluyendo el ejercicio libre de la profesión(47\%), la Secretaría de Salud (26\%), el IMSS (15\%) y otros (12\%). (Cuadro No. 4). Las áreas de asignación corresponden a: 19\% en áreas de Gíneco obstetricia, 17\% en Pediatría, 17\% en Cirugía, $15 \%$ en Urgencias y el resto en Medicina interna, Cuidados intensivos, Consulta externa y Salud Pública. (Cuadro No. 5). Respecto al número de horas semanales laboradas por los egresados se encontró que el $50 \%$ trabaja entre 30 y 40 hrs., el $25 \%$ menos de 30 hrs., y de estos la mitad labora sólo 8 horas. Por otra parte, el 13\% trabaja entre 41 y 48 horas, pero el 12\% refirió de 48 a 60 horas semanales. El 19\% de los entrevistados labora en dos instituciones.

\section{CUADRO No. 4}

Institución contratante de los exalumnos de la ENEO, egresados de la licenciatura en 2003, encuestados a los 3 años de su egreso.

2006

\begin{tabular}{|l|c|}
\hline \multicolumn{1}{|c|}{ Institución } & Porcentaje* $^{*}$ \\
\hline Privados y ejercicio libre & $47 \%$ \\
\hline Secretaría de Salud & $26 \%$ \\
\hline IMSS & $15 \%$ \\
\hline Otras & $12 \%$ \\
\hline
\end{tabular}

Fuente: Misma del cuadro anterior * $\mathrm{n}=41$

\section{CUADRO NO. 5}

Áreas laborales de los exalumnos de la ENEO, egresados de la licenciatura en 2003, encuestados a los 3 años de su egreso.

2006

\begin{tabular}{|l|c|}
\hline \multicolumn{1}{|c|}{ Áreas Laborales } & Porcentaje* \\
\hline Gíneco-Obstetricia & $19 \%$ \\
\hline Pediatría & $17 \%$ \\
\hline Cirugía & $17 \%$ \\
\hline Urgencias & $15 \%$ \\
\hline $\begin{array}{l}\text { Medicina interna, Cuidados intensivos, Consulta } \\
\text { externa y Salud pública. }\end{array}$ & $32 \%$ \\
\hline
\end{tabular}

Fuente: Misma del cuadro anterior * $n=41$
CUADRO NO. 6

Categorías en las que fueron contratados los exalumnos de la ENEO, egresados de la licenciatura en 2003, encuestados a los 3 años de su egreso.

2006

\begin{tabular}{|l|c|}
\hline \multicolumn{1}{|c|}{ Categoría de contratación } & Porcentaje* \\
\hline Auxiliar de Enfermería & $26 \%$ \\
\hline Pasante de Enfermería & $14 \%$ \\
\hline Enfermera general & $26 \%$ \\
\hline Licenciada en Enfermería & $23 \%$ \\
\hline Especialista & $3 \%$ \\
\hline Cargo Administrativo & $3 \%$ \\
\hline Otros & $5 \%$ \\
\hline
\end{tabular}

Fuente: Misma del cuadro anterior * $\mathrm{n}=41$

Las categorías laborales en las cuales estaban contratados fueron: 26\% como auxiliares de Enfermería, 14\% como pasantes, 26\% como Enfermeras generales, 23\% como licenciadas, $3 \%$ como especialistas, $3 \%$ con cargo administrativo y $5 \%$ como otros. El $80 \%$ de los que seguían contratados como pasantes no estaban titulados, mientras que el $88 \%$ de los que se encontraron como licenciadas contaban ya con su título profesional, así como los que fueron contratados como especialistas o con cargo administrativo. Pero el 56\% que ocupan el puesto de auxiliar ya están tituladas y casi todas desempeñan este cargo. Respecto a los tiempos utilizados en el desarrollo de su práctica profesional los egresados afirman que muy frecuentemente o frecuentemente desempeñan:

- Cuidados de Enfermería sencillos tendientes a la resolución de alteraciones patológicas reales o potenciales del enfermo en hospitales (86\% de la población).

- Cuidados de Enfermería complejos tendientes a la resolución de alteraciones patológicas en el enfermo (77\% de la población).

- Cuidados de Enfermería orientados al apoyo integral del paciente y su familia (72\% de la población).

- Cuidados de Enfermería enfocados a la resolución de necesidades fisiológicas del enfermo en hospitales ( 61\% de la población) (Cuadro No. 7).

Solamente el 45\% de los egresados afirma que está realizando con frecuencia actividades educativas orientadas a la prevención y un 57\% reconoce que ofrece orientación dirigida a tratamientos específicos. Por otra parte es importante señalar algunas actividades que los egresados refieren no desempeñar o desempeñar rara vez como: 


\section{CUADRO NO. 7}

Actividades laborales que llevan a cabo muy frecuentemente o frecuentemente los exalumnos de la ENEO, egresados de la licenciatura en 2003, encuestados a los 3 años de su egreso.

2006

\begin{tabular}{|l|c|}
\hline \multicolumn{1}{|c|}{ Actividades laborales } & Porcentaje* \\
\hline $\begin{array}{l}\text { Cuidados de Enfermería sencillos tendientes a la } \\
\text { resolución de alteraciones patológicas reales o } \\
\text { potenciales del enfermo en hospitales. }\end{array}$ & $86 \%$ \\
\hline $\begin{array}{l}\text { Cuidados de Enfermería complejos tendientes } \\
\text { a la resolución de alteraciones patológicas en el } \\
\text { enfermo }\end{array}$ & $77 \%$ \\
\hline $\begin{array}{l}\text { Cuidados de Enfermería orientados al apoyo } \\
\text { integral del paciente y su familia }\end{array}$ & $72 \%$ \\
\hline $\begin{array}{l}\text { Cuidados de Enfermería enfocados a la } \\
\text { resolución de necesidades fisiológicas del } \\
\text { enfermo en hospitales. }\end{array}$ & $61 \%$ \\
\hline
\end{tabular}

Fuente: Misma del cuadro anterior $n=41$

- Actividades de Coordinación de investigación

(el 75\% de la población).

- Actividades de Participación en investigación

(el 75\% de la población).

- Actividades de supervisión

(el 74\% de la población).

- Actividades administrativas de apoyo para el funcionamiento del servicio (el 57\% de la población).

Al cuestionarlos acerca de las actividades para las cuales se sentían más preparados se identificaron las siguientes: cuidados de Enfermería enfocados a la resolución de necesidades fisiológicas en el enfermo (55\%), cuidados de Enfermería complejos tendientes a la resolución de alteraciones patológicas en el enfermo (45\%), cuidados de Enfermería enfocados al apoyo integral del paciente y su familia (45\%) y cuidados de Enfermería sencillos tendientes a la resolución de alteraciones patológicas reales o potenciales (42\%).

Al cuestionarlos sobre las actividades para las que se sentían menos preparados se encontraron las siguientes: activida- des de coordinación de investigación (52\%), actividades de participación en investigación (44\%), actividades administrativas de apoyo para el funcionamiento del servicio (37\%) y actividades de supervisión (37\%). Respecto al factor o factores que obstaculizan el desempeño laboral destacaron: $32 \%$ la falta de experiencia, 32\% la falta de reconocimiento profesional, 27\% la falta de personal, 15\% la falta de material y equipo y $7 \%$ conflictos interpersonales. (Cuadro No. 8). En cuanto a la congruencia que experimentan entre la formación recibida y el mercado laboral, el 49\% la calificaron como "mucha", el 37\% como "regular", el 11\% como "poca" y el 3\% como "nula" (Cuadro No. 9).

\section{CUADRO NO. 8}

Factores que obstaculizan el desempeño laboral de los egresados de 2003, opinión emitida a los 3 años de su egreso

2006

\begin{tabular}{|l|c|}
\hline \multicolumn{1}{|c|}{ Factores } & Porcentaje* \\
\hline Falta de experiencia & $32 \%$ \\
\hline Falta de reconocimiento profesional & $32 \%$ \\
\hline Falta de personal & $27 \%$ \\
\hline Falta de material y equipo & $15 \%$ \\
\hline Conflictos interpersonales & $7 \%$ \\
\hline
\end{tabular}

Fuente: Misma del cuadro anterior ${ }^{*} n=41$

\section{CUADRO NO. 9}

Congruencia que experimentan los egresados de 2003 entre la formación recibida y el mercado laboral, encuestados a los tres años de su egreso.

2006

\begin{tabular}{|l|c|}
\hline \multicolumn{1}{|c|}{ Congruencia } & Porcentaje* $^{*}$ \\
\hline Mucha & $49 \%$ \\
\hline Regular & $37 \%$ \\
\hline Poca & $11 \%$ \\
\hline Nula & $3 \%$ \\
\hline
\end{tabular}

Fuente: Misma del cuadro anterior ${ }^{*} \mathrm{n}=41$ 
En cuanto a la congruencia entre los aprendizajes de las asignaturas obligatorias del plan de estudios y el desempeño de sus actividades profesionales, de acuerdo con los datos de la regresión múltiple entre los 9 enunciados que representan a las actividades en las prácticas del cuidado y las materias tanto obligatorias como las optativas se observó asociación débil, pero con tendencia positiva, a mayor valoración de las actividades de la práctica del cuidado, mayor asociación con la valoración de la aportación que ofrecen las materias obligatorias.

Respecto a la satisfacción laboral de los alumnos el 91\% la calificó afirmativamente y lo justificó primordialmente por la oportunidad de superarse en el ámbito profesional y personal, así como por la complacencia de poder ayudar al otro.

Respecto al "sueldo quincenal" que perciben, se puede mencionar que el $17 \%$ de los reciben menos de $\$ 2000.00$, el $40 \%$ recibe de $\$ 2000.00$ a $\$ 3000.00 ;$ el $17 \%$ más de $\$ 3000.00$ y hasta $\$ 4500.00$ y $26 \%$ de $\$ 4501.00$ hasta $\$ 7000.00$

El 70\% menciona que recibe prestaciones laborales, encontrando con mayor frecuencia: seguro médico (70\%), préstamos (43\%), estímulos económicos (43\%), despensa (37\%), actualización técnica (27\%), y actualización humanística (20\%)

El 97\% expresó su deseo por continuar con su desarrollo profesional, para ampliar sus conocimientos (65\%), ser mejor Enfermera (38\%) y obtener mejor sueldo (26\%). Y los ámbitos de formación demandados fueron: las Especialidades (76\%), la Maestría en Enfermería (21\%) y otras maestrías (15\%), además mencionan el deseo por realizar otros estudios complementárnoslas especialidades más referidas fueron: Enfermería Infantil (32\%), Enfermería Perinatal (20\%), incluyendo Obstetricia y Psicoprofilaxis, Enfermería del Adulto en Estado Crítico (16\%); el resto mencionó diversas ramas como: Enfermería en Salud Pública, Cardiología, Neurología, Docencia y Médico Quirúrgica. Casi la mitad de los egresados manifestó que le gustaría continuar estudiando otra carrera como Medicina, Psicología o Administración, sin embargo ellos mismos expresan que quizá lo que hagan es una especialidad o maestría.

El 57\% de los encuestados mencionó que no recibe estímulos para su desempeño profesional y el $43 \%$ que si los recibe afirma que estos casi siempre son de carácter económico. Respecto a la oportunidad de actualización, el 59\% respondió afirmativamente y las áreas de mayor oferta fueron: manejo de equipos especializados (32\%), técnicas especiales de Enfermería (32\%), cuidados de Enfermería (27\%), resucitación cardiopulmonar (29\%), farmacología (15\%), relaciones humanas (15\%) y promoción a la salud (12\%). En relación a una pregunta orientada a qué le recomendaría el egresado a los profesores para la formación de las nuevas generaciones se encontró lo siguiente; que se propicie la formación en la práctica clínica (63\%), que se actualicen periódicamente (46\%), que promuevan valores a través de las actitudes (32\%) y que establezcan relaciones humanas positivas (29\%). (Cuadro No.10).

\section{CUADRO NO. 10}

Recomendaciones de los egresados en 2003
para los profesores en cuanto a la formación
de nuevas generaciones

2006

\begin{tabular}{|l|c|}
\hline \multicolumn{1}{|c|}{ Congruencia } & Porcentaje \\
\hline Propiciar la formación práctica & $63 \%$ \\
\hline Actualizarse periódicamente & $46 \%$ \\
\hline Promover valores a través de las actitudes & $32 \%$ \\
\hline Establecer relaciones humanas positivas & $29 \%$ \\
\hline
\end{tabular}

Fuente: Misma del cuadro anterior $\mathrm{n}=41$

\section{ANÁLISIS DE LOS RESULTADOS}

La población estudiada en el 2006 correspondió al 31\% de la población que egresó en 2003. La edad promedio encontrada es muy similar a los seguimientos anteriores, persiste la presencia de un grupo de alumnos de mayor edad, ${ }^{4}$ hecho que hay que considerar para crear medidas de apoyo educativo para alumnos con responsabilidades familiares y laborales afines a su grupo etáreo. En contraste con los seguimientos de egresados de 2004 y 2005 la proporción entre hombres y mujeres registra un ligero cambio a favor de las últimas, pero sin alcanzar las cifras de seguimientos anteriores, ${ }^{5}$ la observación de las tendencias subsiguientes permitirá estudiar mejor el fenómeno. Los datos sobre el estado civil son muy similares al seguimiento anterior pero hay un ligero incremento en cuanto a la proporción de exalumnos con hijos y como en 
otras generaciones se evidencia el riesgo de no titulación o de condiciones laborales desfavorables en egresadas con mayor paridad. ${ }^{6}$ En relación a su perfil académico, el promedio de calificación fue ligeramente inferior al de la generación anterior, pero la proporción de alumnos con promedio entre 8.1 y 9 fue mayor. En cuanto a la reprobación se observa un incremento al comparar con cifras anteriores, encontrando casi las mismas asignaturas que en las dos últimas generaciones pero con incremento en Anatomía y Nutrición, cabe mencionar que este grupo de población estaba en sus primeros semestres cuando ocurrió la prolongada huelga universitaria. ${ }^{7}$ y ${ }^{8}$ En cuanto a la opinión sobre la calidad de la formación teórica se mantiene similar al seguimiento anterior, sin embargo la opinión sobre la calidad de la formación práctica desciende, alcanzando cifras más parecidas a las referidas en 2004, ${ }^{9}$ habrá que observar cifras subsecuentes ya que la tendencia desde 2001 era favorable, identificando ésta como un hallazgo satisfactorio en el ámbito de la enseñanza. ${ }^{10}$ En cuanto al nivel de atención en el que se lleva a cabo el servicio social se observa un perfil más parecido a 2004, en esta generación se vuelve a aumentar la proporción de pasantes en áreas de Investigación, hecho que coincide con el impulso de la investigación en Facultades y Escuelas de la UNAM. ${ }^{11}$ La proporción de titulados al término de los 3 años presenta un incremento importante, lo cual irá en beneficio de sus oportunidades laborales. La opción de titulación por EPAC se mantiene con ventaja sobre otras opciones, hecho que responde a políticas institucionales que en busca del incremento de la titulación programan este tipo de exámenes con más regularidad y frecuencia. En cuanto a su inserción en el mercado laboral se encuentra un incremento al comparar con 2005, ${ }^{12}$ sin embargo llama la atención de los egresados que no están laborando quienes prioritariamente lo atribuyen a la falta de oportunidad en el mercado laboral, situación que no se había evidenciado en otros seguimientos. La contratación de los egresados por instituciones privadas sigue tomando ventaja, fenómeno que los centro formadores deben identificar para poder orientar en forma pertinente. En el caso del IMSS y de la Secretaría de Salud se observa un ligero repunte a expensas de la ausencia de contrataciones referidas en el ISSSTE. Continúa el descenso en cuanto a la asignación en áreas de cuidados intensivos, en cambio se observa la presencia importante de asignaciones en Gíneco-obstetricia y Pediatría, lo que puede estar respondiendo a políticas específicas gubernamentales. ${ }^{13} \mathrm{El}$ número de horas contratadas presenta variabilidad, pero con un patrón similar al de otros seguimientos, sin embargo en esta generación se pudo constatar que casi una quinta parte labora en dos instituciones, lo que responde a los bajos salarios y a la pérdida de poder adquisitivo.

En cuanto a las categorías de contratación se observa una disminución en el caso de Enfermera general, pero un aumento para las categorías de licenciada, especialista y cargo administrativo, en cuanto a la asignación de las egresadas en estas tres últimas categorías, a excepción de una, contaban con el título profesional; en contraste con las egresadas con categoría de pasantes, quienes carecían del título profesional en su mayoría, por lo que una vez más se confirma la asociación del título profesional con una mejor oportunidad laboral, por lo que la Escuela de Enfermería debe continuar con la promoción de las diversas opciones para la titulación. En cuanto a la frecuencia con la cual desempeñan sus actividades, los cuidados de Enfermería sencillos y complejos orientados a la resolución de alteraciones en el enfermo, así como los cuidados enfocados al apoyo integral continúan ocupando un lugar prioritario como en los seguimientos de años anteriores, lo cual coincide con el perfil esperado, sin embargo llama la atención que un poco más de la mitad de las egresadas no refiere la realización de actividades educativas orientadas a la prevención como actividades frecuentes, cuando las políticas y la realidad nacional si lo demandan. ${ }^{14}$

En cuanto a las áreas de mayor competencia experimentada por los alumnos, una vez más señalan aquellas que desarrollan más. Por otra parte, las actividades para las que refieren tener menor competencia, como son las de investigación y de gerencia, son actividades que podrán ir reforzando con el desarrollo profesional subsecuente, en las especialidades y en la maestría. La identificación de la falta de experiencia como obstáculo para su desempeño, sigue siendo frecuente, hecho que concuerda con la opinión de los egresados sobre la necesidad de tener mejor formación práctica, aspecto que habrá de seguir considerándose para la planeación y operación de las prácticas clínicas.

La falta de reconocimiento profesional se refiere en mayor proporción, lo cual debe preocupar al gremio y a las instituciones formadoras de recursos, buscando estrategias que mitiguen este problema. Respecto a las carencias de personal, así como, de material y equipo, aún cuando no dependen de los centros educativos si demandan una alerta profesional ya que ambas van en detrimento de la oferta de servicios de Enfermería de calidad y por lo tanto de seguridad del paciente, rubro al que la OPS está dando relevancia actualmente. ${ }^{15}$ Mejora la percepción de la congruencia entre la formación y el mercado al compararla 
con los seguimientos anteriores, 16 y 17 habrá que seguir valorando y apoyando ya que un desempeño favorable, ofrece mayor satisfacción personal.

Como en años anteriores los egresados siguen manifestando su interés por continuar su formación en el posgrado, en las especialidades más que en la maestría, lo que puede explicarse por el campo de acción en el ámbito laboral y por el reconocimiento institucional de muchas de éstas en el mercado con su consecuente compensación salarial.

Sin embargo es importante destacar el incremento en la proporción de egresados que buscarán su crecimiento profesional a través de la Maestría en Enfermería, ${ }^{18}$ lo cual responde a un mayor conocimiento de ésta, pues ya egresó la primera generación.

En cuanto a las especialidades que mencionan con mayor frecuencia, se observa un repunte en Enfermería Perinatal y una disminución en Cuidados Críticos ( Adulto en Estado Crítico); y se mantiene la Enfermería Infantil; estas orientaciones coinciden con las áreas en las cuales están asignadas principalmente, lo cual favorece potencialmente su ingreso a través de convenios con las instituciones en las que laboran. Como en otros seguimientos, la mayoría de los egresados manifiestan que no reciben estímulos y cuando los reciben mencionan que no son de carácter académico, sin embargo la mayoría aceptan que tienen oportunidad de actualizarse. En cuanto a las recomendaciones que los exalumnos proponen a sus profesores, al mejoramiento de la formación práctica, sin dejar de mencionar la actualización continua de sus profesores y la manifestación de actitudes humanas, recomendaciones que tanto la escuela como los profesores deben tener presente en el ejercicio diario de su docencia para favorecer la formación de profesionistas que la sociedad actual exige: científica y tecnológicamente preparados y humanamente desarrollados.

\section{CONCLUSIONES}

El perfil sociodemográfico de la población escolar debe ser un factor por considerar en los centros formadores de recursos para mejorar la eficiencia terminal. Aún cuando la dependencia establece medidas remediales para las materias de mayor reprobación, todavía no se abaten. Sigue presente la demanda de los egresados respecto la necesidad de mejorar la enseñanza en la práctica clínica.

La contratación y la ocupación de mejores categorías continua siendo favorecida con la titulación oportuna, aspecto que la Escuela y la Universidad continúan reforzando. Persiste el predominio del sector privado en la contracción laboral, motivando a los centros educativos a la formación de profesionistas científica y tecnológicamente capacitados, así como humanamente preparados para responder a las demandas de un mundo globalizado pero sin menoscabo de la obtención de una fuerte identidad profesional.

Continúa el predominio en las actividades orientadas a la curación con desventaja para las actividades de prevención, sin las cuales el sistema de salud no podrá darse abasto. La tendencia positiva en la asociación entre la formación académica y las actividades de la práctica del cuidado propiciarán una mayor congruencia entre la formación, y el desempeño en la práctica, lo que permitirá subsanar los fuertes impactos económicos, políticos y culturales de la globalización.

La identificación del cuidado orientado al apoyo integral como uno de los que ocupan altas proporciones de sus horas laborales encamina a la profesionalización idónea de la Enfermera. El deseo de actualización profesional de los egresados propiciará el desarrollo de la profesión y coadyuvará para los reconocimientos económicos.

La proporción de desempleo es baja al compararla con otras áreas, pero la doble jornada y la baja remuneración son factores de alta preocupación para el gremio.

\section{REFERENCIAS BIBLIOGRÁFICAS}

1 Bolsa Universitaria de trabajo y Dirección General de Planeación. Dos estudios sobre egresados de la UNAM. Cuadernos de Primaria Universitaria 3a copia, año 1988.

2 Legislación Universitaria. Estatuto General de la UNAM

3 Rubio Domínguez, Severino. Informe de Dirección 2003 ENEO UNAM

4 Müggenburg, C y cols. Seguimiento de los egresados de la Licenciatura en Enfermería de la ENEO UNAM en 2005 Biblioteca ENEO UNAM

5 Müggenburg ,C. Barreto Plácido, F y García Ortiz, R. Seguimiento de egresados de la Licenciatura en Enfermería de la ENEO en 2000 a los tres años (2003) Enfermería Universitaria Vol.3 No. 1 Año 3 Enero-Abril 2006

6 Müggenburg, C y cols. Seguimiento de los egresados de la Licenciatura en Enfermería de la ENEO UNAM en 2004 Biblioteca ENEO UNAM

7 y 8 Müggenburg Rodríguez, C. Pérez Cabrera,I. Ramírez Toriz, L. Castañeda Godínez, C. García Sánchez, E. Seguimiento de los 
egresados de la ENEO UNAM 2004 Metamorfosis en Enfermería Vol. 1 No.4 Mayo Junio 2006 pp. 5-10 y Müggenburg, $C$ y cols. Seguimiento de los egresados de la Licenciatura de la ENEO UNAM en 2005 Biblioteca ENEO UNAM

9 Müggenburg, C. y cols. Seguimiento de los egresados de la Licenciatura en Enfermería de la ENEO UNAM en 2004 Biblioteca ENEO UNAM

10 Müggenburg, C. y cols. Seguimiento de los egresados de la Licenciatura de la ENEO UNAM en 2005 Biblioteca ENEO UNAM

11 Legislación Universitaria UNAM Artículo 8 (Acuerdo del Consejo Universitario en Junio de 2003)

12 Müggenburg, C. y cols. Seguimiento de los egresados de la Licenciatura de la ENEO UNAM en 2005 Biblioteca ENEO UNAM

13 Cahuana-Hurtado L, Sosa-Rubí S, Bertozzi S. Costo de la atención materno infantil en el estado de Morelos, México.

Salud Publica Mex 2004;46:316-325. El texto completo en inglés de este artículo está disponible en: http://www.insp.mx/salud/index.html 8/agosto/2007

14 Secretaría de Salud. Programa Nacional de Salud 2007-2012 (Estrategia 2)

15 La OMS lanza "Nueve soluciones para la seguridad del paciente" a fin de salvar vidas y evitar daños Oficina de Información Publica, OMS Ginebra - Suiza. 02 de mayo de 2007

http://www.bol.ops-oms.org/servicios/?DB=B\&S1 1 =12515\&SE=SN (Tomado el 8 de agosto de 2007)

16 y 17 Müggenburg, C. y cols. Seguimiento de los egresados de la Licenciatura en Enfermería de la ENEO UNAM en 2004 Biblioteca ENEO UNAM y Müggenburg, C. y cols. Seguimiento de los egresados de la Licenciatura de la ENEO UNAM en 2005 Biblioteca ENEO UNAM

18 Müggenburg, C. y cols. Seguimiento de los egresados de la Licenciatura de la ENEO UNAM en 2005 Biblioteca ENEO UNAM

Bolsa Universitaria de trabajo y Dirección General de Planeación. Dos estudios sobre egresados de la UNAM. Cuadernos de Primaria Universitaria 3a copia, año 1988.

Legislación Universitaria. Estatuto General de la UNAM. México: UNAM, 2005:

Cahuana-Hurtado L, Sosa-Rubí S, Bertozzi S. Costo de la atención materno infantil en el estado de Morelos, México.

Rubio Domínguez, Severino. Informe de Dirección 2003 ENEOUNAM. México: ENEO, 2004

Navarro Leal, Marco Antonio. En ANUIES Esquema básico para estudios de egresados. México: Biblioteca de la Educación Superior. Serie Investigaciones 2003. p. 11.

Müggenburg C y cols. Seguimiento de los egresados de la Licenciatura en Enfermería de la ENEO UNAM en 2003. Enfermería Universitaria 2006; 3(1); 5-13
Müggenburg, Rodríguez Ma. Cristina, Pérez Cabrera Iñiga, Ramírez Toriz Leticia, Castañeda Godínez Cristina y García Sánchez Ma. Elena. "Seguimiento de los egresados de la Licenciatura en Enfermería de la ENEO UNAM en 2004" Metamorfosis en Enfermería; 1(4); 5-10.

Informes técnicos de los seguimientos de egresados de la ENEO análisis. Biblioteca ENEO (Catálogo en www.eneo.UNAM. $\mathrm{mx}$ /comitedeinvestigación).

La OMS lanza "Nueve soluciones para la seguridad del paciente" a fin de salvar vidas y evitar daños, Oficina de Información Publica, OMS Ginebra - Suiza. 02 de mayo de 2007

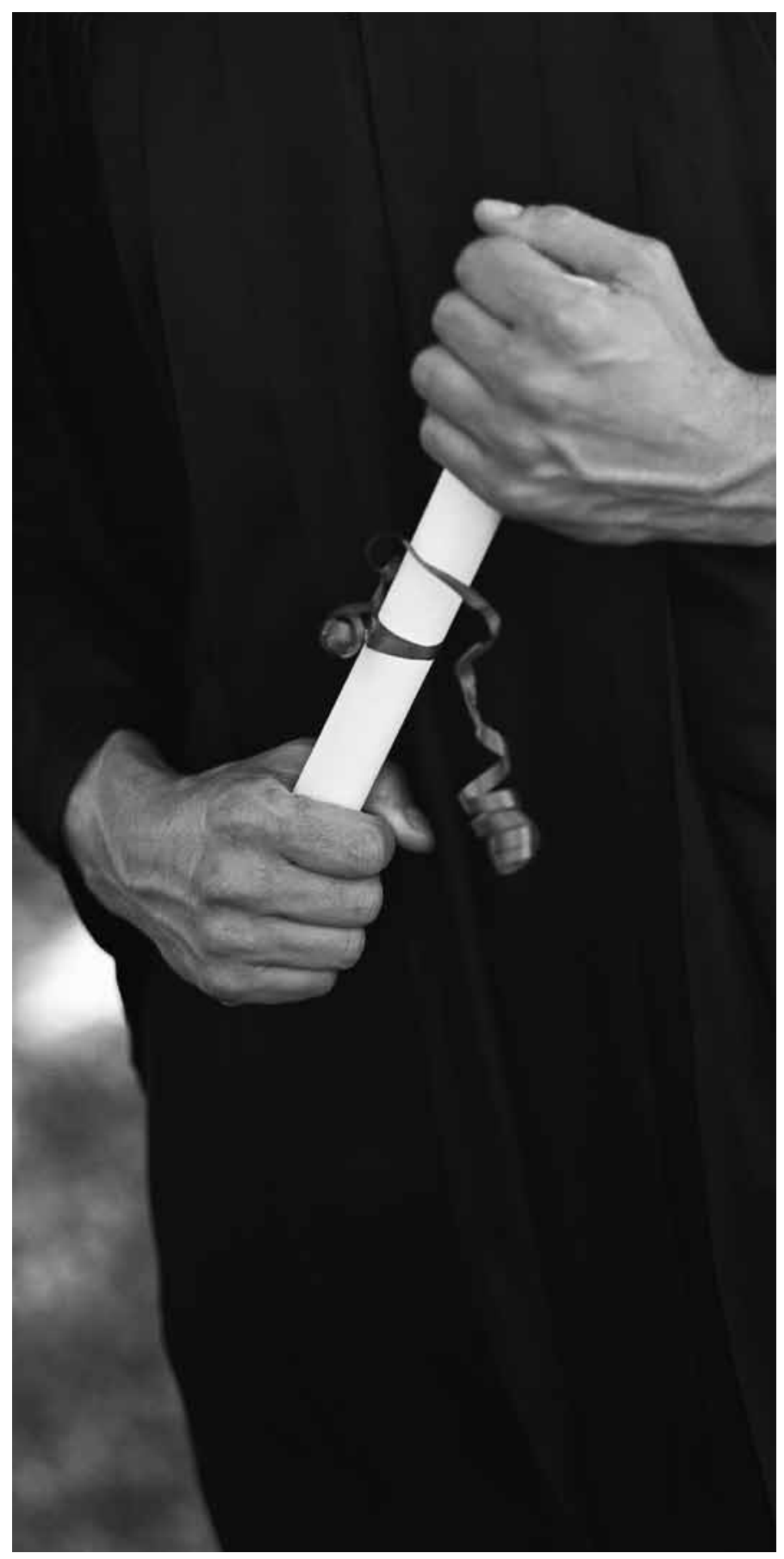

